\title{
Identificação, seleção e caracterização de espécies vegetais destinadas à instalação de jardins sensoriais táteis para deficientes visuais, em Piracicaba (SP), Brasil( ${ }^{(1)}$
}

\author{
JOSÉ FLÁVIO MACHADO CÉSAR LEÃO(1)
}

\begin{abstract}
RESUMO
O trabalho avaliou a percepção tátil de espécies vegetais por uma parcela da população de deficientes visuais em diferentes graus de intensidade, de ambos os sexos e com idades entre 13 e 77 anos, residentes em Piracicaba (SP), classificando-as de acordo com a maior ou a menor preferência, por meio de análises sensoriais afetivas. As plantas foram escolhidas dentre aquelas utilizadas na composição de parques e jardins, instalados nas condições ambientais da região de Piracicaba (SP). No processo de seleção das plantas, considerou-se, também, a escolha daquelas que possibilitassem maior segurança física e psicológica aos deficientes visuais, além da sua disponibilidade no mercado. Selecionaram-se, para os testes, indivíduos vegetais adultos, organizados em grupos distintos, de acordo com seu porte, sua estrutura e suas funções específicas na composição paisagística: 13 espécies arbóreas; cinco palmeiras; dois tipos de bambus; 19 arbustos, 34 espécies herbáceas e quatro tipos de gramas, totalizando 77 diferentes plantas. As espécies arbóreas foram avaliadas pelas características do tronco: a circunferência à altura do peito (CAP) e a textura da casca; as arbustivas, segundo o porte da planta, o tipo, a textura e o tamanho das folhas; as herbáceas e as gramíneas, segundo o tipo, a textura e o tamanho das folhas. A partir dos testes realizados, concluiu-se que, com relação aos provadores, não houve diferença significativa entre os fatores sexo, idade, grau e tempo da deficiência, no que se refere às preferências sobre as espécies vegetais, dentro de cada categoria. Em relação às espécies arbóreas, constatouse que, aos menores valores de CAP e à textura mais fina da casca, foram associados os maiores escores médios, refletindo a preferência dos provadores por indivíduos com menores diâmetros e por espécies com texturas macias, lisas e muito lisas. Os resultados sugeriram que a característica textura da casca foi mais importante, do ponto de vista afetivo, que a circunferência do caule à altura do peito. No que se refere aos arbustos testados, verificou-se que o porte foi pouco importante e que a textura e o tamanho das folhas foram fatores preponderantes na discriminação das espécies, com ampla vantagem para as texturas macias. Os testes com as herbáceas e as gramíneas para relvados também evidenciaram que as características mais importantes, do ponto de vista afetivo, foram a textura e o tamanho das folhas. As herbáceas contempladas com flores ou inflorescências receberam escores médios maiores, exceção feita ao antúrio e à estrelícia, provavelmente, por apresentarem folhas médias e grandes, respectivamente, ou pelas características específicas de suas flores.
\end{abstract}

Palavras-chave: jardins sensoriais; deficientes visuais; cegos; tato; inclusão social; plantas táteis.

\section{ABSTRACT \\ Identification, selection and characterization of plant species designed to establish tactile sensory gardens for the vision impaired, in Piracicaba (SP), Brazil}

\begin{abstract}
The tactile perception of plant species by a visual impaired population of Piracicaba (SP) were evaluated at different impair intensity degree, gender and age, assorted according to higher or lower preference through affective sensorial analysis. The plants were collected from parks and gardens under the environmental conditions of Piracicaba (SP). The selection of plants for this study considered physical and psychological safety to the visual impaired and market availability. Adult plants were selected for the tests, organized into distinct groups according to size, structure and specific function in the landscape composition: thirteen arboreal species; five palm trees; two types of bamboo; nineteen shrubs; thirty four herbaceous species and four types of grass, in a total of seventy seven different plants.

The arboreal species were evaluated according to the trunk characteristics: circumference at breast height $(\mathrm{CBH})$ and bark texture; shrubby plants according to plant size, type, texture and leaf size; herbaceous plants and grasses according to type, texture and leaf size. Regarding the testers, the results showed that no significant difference occurred among gender, age, deficiency degree and time as to the preference for plant species within each category. For arboreal species, one verified that lower $\mathrm{CBH}$ values and thinner bark texture were associated to the mean scores, thus reflecting the preference of testers for lesser diameters and soft, smooth and very smooth texture species. The results suggested that the bark texture feature was more important, from the affective viewpoint, than the stem circumference at breast height. Regarding the tested shrubs, one verified that the size was not significant and that both leaf texture and size
\end{abstract}

\footnotetext{
(1) Parte da Tese de Doutorado do autor apresentada à Escola Superior de Agricultura "Luiz de Queiroz" (Esalq-USP). Recebido para publicação em 4/7/2007 e aceito em 25/7/2008.

(2) Departamento de Produção Vegetal (Fitotecnia). Esalq-USP. Caixa Postal 9. 13418-900 Piracicaba (SP).
} 
were preponderant in discriminating the species, with soft textures being much more preferred. Tests with herbaceous plants and grasses for grassland also pointed that the more important characteristics, from the affective viewpoint, were leaf texture and size. Blooming herbaceous plants were given higher mean scores, except for the Anthurium and Strelitzia, probably because of their medium and large size leaves, respectively, or due to specific flower features.

Keywords: sensory gardens; visual handicaps; blind people; touch; social inclusion; landscape history; tactile plants

\section{INTRODUÇÃO}

Os jardins sempre foram, através dos tempos, uma inesgotável fonte de prazer e de recreação para as pessoas de todas as idades, nas mais diversas regiões do planeta, desde os primórdios das civilizações. Essa importância foi lembrada, inclusive, na Bíblia, que descreveu os jardins do Éden como um paraíso, verdadeiro presente de Deus aos homens, que se perdeu, após Adão e Eva terem cometido o pecado original.

Ao longo do tempo, os jardins acompanharam o desenvolvimento dos diversos povos - babilônios, egípcios, gregos, romanos e árabes. Durante a Renascença, alcançaram grande esplendor, principalmente, na França e na Itália. Mais tarde, ganharam novas características na Inglaterra, com a destinação de grandes áreas para a formação de parques com aspecto mais natural.

Recentemente, os parques públicos e particulares, sobretudo nos países desenvolvidos, têm se tornado importantes locais de visitação, onde o homem moderno tem a possibilidade de se reencontrar com a natureza, de recuperar as energias perdidas, de livrar-se do estresse e das preocupações da atribulada vida atual. Verifica-se, no entanto, que uma expressiva parcela da população ainda fica à margem dessa importante fonte de lazer e de recreação: os deficientes visuais.

No Brasil, a falta de informações aprofundadas torna o problema mais acentuado, pois, além do restrito conhecimento técnico sobre o tema, pouco se tem investido em iniciativas voltadas para esse público. Assim, de forma geral, toda a teoria empregada no planejamento dos jardins leva em conta valores estéticos, cujos elementos privilegiam principalmente o sentido da visão. A forma, a cor, a textura das plantas não facilita e, muitas vezes, dificulta a percepção pelos deficientes visuais.

O Instituto Brasileiro de Geografia e Estatística (IBGE) mensurou, pela primeira vez, no recenseamento de 2000, o número de portadores de deficiências físicas e constatou que $9,8 \%$ da população brasileira, ou seja, 16,6 milhões de pessoas têm algum tipo de deficiência visual.

Desse número, quase 159 mil são privados da visão (cerca de 1\% da população), 2,4 milhões possuem grande dificuldade permanente de enxergar e cerca de 14 milhões têm alguma dificuldade permanente de enxergar. A maior parte dos deficientes visuais habita o Sudeste do País: 59.238 pessoas, seguida de perto pela região Nordeste (52.689). Os demais encontram-se no Centro-Oeste (16.525), no Norte (10.414) e somente 6.991 pessoas residem no Sul.

Por outro lado, considera-se fundamental que as oportunidades oferecidas aos deficientes visuais abranjam todas as áreas, tais como trabalho, educação, saúde, lazer, esporte, entre outras. Nesse sentido, os parques e os jardins podem constituir-se em importantes elementos de aprendizagem, de convivência, de terapia e, principalmente, de inclusão social.

Apesar da existência de jardins construídos para atender especificamente a esse tipo de público, em vários pontos do mundo, tais iniciativas são, ainda, incipientes nos países em desenvolvimento, como o Brasil. Sabe-se que são poucos os espaços verdes planejados para deficientes visuais no País, com destaque para aqueles instalados no Jardim Botânico do Rio de Janeiro e no Parque do Trote, em São Paulo.

Observa-se que pouco se conhece, ainda, sobre a vegetação mais adequada a empregar na criação de projetos do gênero, com capacidade de sensibilizar, de forma efetiva, o público portador de deficiência visual, em especial, levando-se em conta a grande diversidade de espécies vegetais existentes no Brasil.

Dessa forma, o presente trabalho pretendeu identificar, selecionar, comparar e avaliar, dentre as diversas categorias de plantas (árvores, arbustos, herbáceas, trepadeiras e relvados) utilizadas, normalmente, na composição paisagística de jardins na região de Piracicaba, no Estado de São Paulo, aquelas mais adequadas para o emprego em jardins sensoriais, do ponto de vista tátil.

O estudo levou em conta os seguintes fatores: a adaptação das espécies vegetais às condições edafoclimáticas locais; sua importância como elemento na composição paisagística do jardim; as funções específicas de cada categoria de plantas e sua capacidade para atender às necessidades e às expectativas dos usuários e, finalmente, a disponibilidade de aquisição.

Para a seleção das plantas considerou-se, também, critérios como forma, dimensão (porte adulto), textura, aspectos da folhagem, floração e frutificação, que sejam atraentes e estimulem o tato - pois as mãos funcionam como verdadeiros "olhos" para os deficientes visuais -, possibilitando-lhes desfrutar dos espaços ajardinados com segurança e vivenciar experiências altamente enriquecedoras, em maior contato com a natureza.

Foram analisados, ainda, alguns fatores que limitam o uso de determinadas espécies vegetais nos jardins sensoriais táteis para deficientes visuais, como presença de espinhos, acúleos, folhas pontiagudas, ramos agressivos, partes urticantes, lactíferas, ou que causem alergias; espécies que atraiam insetos e animais peçonhentos; apresentem frutos grandes - com perigo de queda, raízes tabulares, ou ainda pneumatófaros - que dificultam o acesso dos deficientes até às plantas, ou, mesmo, ramos quebradiços ou de inserção muito baixa no tronco, entre outros.

As espécies vegetais selecionadas para uso em jardins sensoriais, no total de 77 representantes vegetais 
distintos, foram submetidas a testes de aceitabilidade por um grupo de deficientes visuais, em diferentes graus de intensidade, com idades variadas, no período de agosto a dezembro de 2006, em parques, jardins e viveiro especializado, todos situados na região de Piracicaba (SP).

\section{MATERIAL E MÉTODOS}

\subsection{Material}

\subsubsection{Seleção das espécies vegetais}

Dentre a diversidade de espécies vegetais utilizadas nos projetos de paisagismo, selecionaram-se para estudo apenas aquelas perfeitamente adaptadas às condições ambientais da região de Piracicaba (SP). Outro fator levado em conta foi a disponibilidade dessas plantas ornamentais no mercado.

A seguir, organizaram-se essas espécies vegetais em grupos distintos, considerando-se não apenas seu porte e estrutura, como também as características específicas da sua arquitetura, que determinam suas funções na composição em um parque ou jardim, a saber: árvores; palmeiras; bambus; espécies arbustivas; espécies herbáceas; trepadeiras e gramíneas utilizadas para a formação de relvados.

\subsubsection{Espécies vegetais descartadas}

$\mathrm{Na}$ seleção da vegetação a analisar, foram desqualificadas para o estudo as plantas que pudessem representar riscos à segurança física e psicológica dos deficientes visuais, por ocasião da realização dos testes, e também no caso de uso público nos locais ajardinados.

A seguir, os critérios utilizados para a exclusão de determinadas espécies vegetais:

- Plantas com espinhos e acúleos que poderiam causar ferimentos, como Erytrina sp. (suinã), Bougainvillea sp. (primavera) e a maioria das cactáceas.

- Plantas com frutos grandes e pesados, que poderiam causar problemas com sua queda: Lecythis pisonis (sapucaia) e Dilenia indica (árvore-das-patacas).

- Plantas com raízes tabulares ou pneumatóforos, nas quais os deficientes visuais poderiam tropeçar: árvores do gênero Ficus sp. (figueiras), Delonix regia (flamboiã); Taxodium distichum (pinheiro-do-brejo).

- Plantas de grande porte com estrutura frágil ou com folhas muito volumosas, que poderiam cair sobre os usuários dos jardins: Schyzolobium parahyba (guapuruvu), porque possui ramos quebradiços; palmeiras do gênero Roystonea (palmeiras imperial e real), que atingem porte muito elevado e têm folhas pesadas.

- Árvores que poderiam causar intoxicações, alergias ou prejudicar a saúde, a exemplo de Nerium oleander (espirradeira) e Schinus molle (aroeira-salsa).

- Plantas com seiva causticante, que poderiam causar problemas dermatológicos: diversas espécies do gênero Euphorbia, como Euphorbia caracasana (vinagreira).

- Plantas com folhas cortantes, que poderiam ocasionar ferimentos nos usuários: Cortaderia selloana (capim-dospampas) e Melissa officinalis (erva-cidreira).

- Plantas que atraíam e abrigavam insetos ou animais peçonhentos, que poderiam oferecer qualquer tipo de perigo, a exemplo de Tecoma stans (ipê-de-jardim) e Triplaris brasiliana (pau-formiga).

\subsubsection{Espécies vegetais estudadas}

Com o objetivo de avaliar as preferências em termos afetivos, oriundos da percepção tátil de deficientes visuais, no tocante aos componentes de jardins sensoriais, foram selecionados, prévia e estrategicamente, 77 representantes vegetais distintos (tabela 1). Tais plantas foram selecionadas dentre aquelas normalmente utilizadas na composição de jardins de uso público e particular, enfatizando quatro categorias: arbóreas, arbustivas, herbáceas e gramas com, respectivamente, 20, 19, 34 e 4 espécies. Dentre as arbóreas, foram tomadas 13 espécies de árvores propriamente ditas, cinco de palmeiras e duas de bambus.

A organização botânica da nomenclatura e a classificação das espécies vegetais estudadas foram realizadas por meio da comparação com fotos disponíveis na literatura pertinente. Para tanto, utilizaram-se os trabalhos de LORENZI (1992, 2002), LORENZI e SOUZA (2001) e LORENZI et al. (2003). É importante ressaltar que alguns nomes científicos das espécies vegetais foram alterados, de acordo com novas sistemáticas propostas tanto por Cronquist, em 1981, como pelo sistema APG II (versão 2003), conforme CAPPELARI JUNIOR (2006).

\subsection{Métodos}

\subsubsection{Análise sensorial}

No planejamento inicial, considerou-se que o experimento deveria contar com um número mínimo de atributos suficientes para a avaliação de suas preferências táteis, dado o tipo de deficiência apresentado pelos provadores. Nesse contexto, as características sensoriais de interesse, selecionadas, a priori, segundo os objetivos deste estudo, apresentaram pequena variação, de acordo com a categoria das plantas utilizadas. As espécies arbóreas foram avaliadas pelas características do tronco: a circunferência à altura do peito (CAP) e a textura da casca; as arbustivas, segundo o porte da planta, o tipo/textura e o tamanho das folhas; as herbáceas e as gramíneas, segundo o tipo/textura e o tamanho das folhas.

Além disso, permitiu-se que a rigorosa classificação acadêmica, observada nos textos clássicos de botânica, fosse traduzida em termos bem mais simples e de fácil compreensão para provadores tão especiais. De modo geral, o perfil sensorial foi desenvolvido pelo método da Análise Descritiva Quantitativa (ADQ), adaptado de MEILGAARD et al. (1999) e STONE e SIDEL (2004).

\subsubsection{Recrutamento e seleção da equipe de provadores}

A equipe de provadores foi constituída de deficientes visuais, em diferentes graus de intensidade, de ambos os sexos e com idades entre 13 e 77 anos. Todos os provadores eram, durante o período experimental, freqüentadores e/ou alunos dos cursos oferecidos pela organização não-governamental Avistar - Associação de Assistência aos Portadores de Necessidades Especiais Visão, de Piracicaba (SP). 
Foi realizado, com os provadores, um treinamento prévio, durante aproximadamente um mês, nas dependências da Avistar, em que se forneceu noções básicas sobre plantas, sua morfologia, sua fisiologia e sua importância, além de conceitos básicos sobre jardinagem e paisagismo. Ao final, foram selecionados 13 provadores que demonstraram possuir disponibilidade, interesse e habilidade para participar dos testes sensoriais.

\subsubsection{Avaliação sensorial das espécies vegetais}

A avaliação sensorial das espécies vegetais foi feita por meio de testes afetivos, verificando-se diretamente a opinião dos provadores. Tais testes constituem uma importante ferramenta, pois acessam diretamente a opinião (preferência e/ou aceitabilidade) do consumidor e/ou o potencial de um produto, com base em características específicas. Como o objetivo do experimento foi avaliar diretamente o grau com que provadores gostam ou desgostam de cada planta incluída na pesquisa, e não o de apontar a planta preferida, os testes ficaram caracterizados como de aceitabilidade.

Além disso, segundo as definições de Damásio e Silva, 1996; Meilgaard et al., 1989 e 1991, citados em FERREIRA (2000), no presente estudo, realizaram-se testes do tipo afetivo-qualitativos, que medem as respostas subjetivas de um pequeno subgrupo de provadores sobre as propriedades sensoriais do objeto; neste caso particular, as espécies vegetais, por meio da verbalização de seus sentimentos e de suas opiniões sobre o objeto.

Dessa forma, em entrevistas individuais, foram apuradas as respostas subjetivas do grupo de provadores sobre as propriedades sensoriais táteis das espécies vegetais previamente selecionadas, por meio da verbalização de seus sentimentos e de suas opiniões sobre as plantas analisadas. O objetivo dos testes foi conhecer o status afetivo dos provadores, com relação às espécies vegetais usadas no paisagismo, utilizando-se, para tanto, a escala hedônica ${ }^{3}$, segundo a adaptação de STONE e SIDEL (2004).

\subsubsection{Desenvolvimento da terminologia descritiva}

Os testes, cada um com duração aproximada de quatro horas, foram realizados no período de agosto a dezembro de 2006, por meio de excursões feitas com o grupo de provadores nos seguintes locais: parque existente na Escola Superior de Agricultura "Luiz de Queiroz" da Universidade de São Paulo, em Piracicaba (SP), situada na Avenida Pádua Dias, 11; no estabelecimento denominado Félix Comércio de Mudas - Rodovia Piracicaba-Limeira, km 119, Limeira (SP) e na sede da Avistar, Rua Liberato Macedo, 847 - Piracicaba (SP).

Inicialmente, atribuiu-se às plantas a serem examinadas, um descritor - termo que descreve uma característica, atributo ou propriedade sensorial de um objeto ou produto. Em seguida, as diversas espécies vegetais foram apresentadas aos provadores, por meio de toque dirigido, nas condições reais de desfrute caracterizadas por espécies plantadas em jardins ou em vasos, sendo-lhes solicitado que submetessem as plantas ao conhecimento tátil.

\subsubsection{Descritores utilizados para árvores, palmeiras e bambus}

No caso das árvores, os provadores avaliaram a rugosidade do tronco, caracterizada pela textura da sua casca e pela presença de ritidomas à altura do peito, nos limites da extensão de seus membros superiores, quando em posição horizontal. As palmeiras e os bambus foram avaliados com relação à forma, à dimensão e à textura do estipe e do colmo, respectivamente, também à altura do peito.

\subsubsection{Descritores utilizados para arbustos, herbáceas e gramíneas}

No caso das espécies arbustivas, herbáceas e gramíneas, os testes incluíram a avaliação das dimensões, das formas, da textura, da consistência de ramos, das folhas e flores (quando disponíveis), além da arquitetura da planta, quando possível, em função de suas dimensões, pois se fossem muito grandes, impossibilitariam o conhecimento da espécie como um todo. Observaram-se outros parâmetros como forma, bordo, consistência e textura das folhas.

Os testes de aceitabilidade foram realizados para se conhecer o status afetivo dos provadores, em relação às espécies vegetais selecionadas, utilizando-se, para tanto, a escala hedônica, que expressa o "gostar" e/ou o "desgostar" em análise sensorial, conforme as normas da ABNT (1993) e a adaptação de STONE e SIDEL (2004).

A escala hedônica foi estabelecida de forma balanceada, com igual número de categorias positivas e negativas, distribuídas nos seguintes conceitos afetivos: gostei muito; gostei; não gostei nem desgostei; desgostei e desgostei muito. Tais conceitos foram associados a classes numéricas. Os escores ${ }^{4}$ utilizados na análise das respostas corresponderam aos pontos médios dessas classes, conforme descrito na Tabela 2.

Cada provador exprimiu individualmente suas opiniões e seus sentimentos, por meio da análise tátil, diante de todas as amostras de plantas selecionadas que lhes foram apresentadas de forma seqüencial. Procurouse evitar qualquer tipo de indução externa do provador envolvido, com relação às amostras estudadas da vegetação.

\subsubsection{Análise estatística}

As respostas obtidas foram analisadas pelas técnicas da análise da variância univariada não-paramétrica de dados desbalanceados e da análise de componentes principais, utilizando-se os sistemas estatísticos SAS (2000) e UNISTAT (2001).

A análise da variância de dados desbalanceados foi desenvolvida conforme CONOVER (1980), IEMMA

$\left(^{3}\right)$ Escala hedônica: do grego $h c ̧ d o n c ̧$, que pode ser traduzido como "prazer". Foi desenvolvida por JONES et al. (1955) e sedimentada por PERYAM e PILGRIM (1957), com o objetivo de medir a aceitação de materiais e produtos. Geralmente, contém cinco (como neste texto), sete ou nove afirmações do entrevistado, caracterizando o "prazer" obtido na prova.

$\left({ }^{4}\right)$ Escore: Valor numérico que traduz, na forma quantitativa, os valores das escalas qualitativas, entre elas a escala hedônica. Neste texto, foram atribuídos valores intervalares entre zero e 10,0, passo 2, e os escores foram tomados como seus pontos médios. 
(1995), CLAUSTRIAUX e IEMMA (1999), IEMMA e CLAUSTRIAUX (1999) e SANTANA e IEMMA (2002).

Para as comparações múltiplas das médias ajustadas dos escores, optou-se pelo critério não paramétrico de DUNN (1964) e, para a comparação entre as medianas, o teste clássico de medianas com probabilidade exata de Fisher, descrito em ZAR (1999). A análise de componentes principais foi realizada segundo FERRARI e IEMMA (1991), PALM (1998) e STONE e SIDEL (2004).

Adotou-se, neste estudo, o nível de significância a = 0,05. Assim, foram considerados significativos os parâmetros ou as funções lineares de seus níveis, para os quais resultaram $\mathrm{p}$-valores tais que $\mathrm{p} £ 0,05$. Os procedimentos da inferência foram precedidos por testes de verificação das pressuposições do modelo: o teste de Dixon-Grubbs-Neuman, para dados discrepantes ou outliers, o teste de Kolmogorov-Smirnov, para normalidade, e o teste de Cochran, para homogeneidade de variâncias, por permitir comparações com variâncias nulas, que ocorrem quando todos os escores são iguais para uma mesma planta.

\section{RESULTADOS E DISCUSSÃO}

\subsection{Performance dos provadores}

Em face das condições especiais dos provadores, a porcentagem média de respostas úteis por provador $(86,7 \%)$, obtidas durante todo o experimento, isto é, na avaliação das 77 espécies vegetais, foi considerada muito boa, apresentando valores médios minimax para provadores $(43,6 \% ; 100,0 \%)$ e para espécies $(79,9 \%$; $92,3 \%)$.

Considerando as condições heterogêneas das características dos provadores, no que se refere aos diversos fatores (sexo, idade, grau e tempo de deficiência), foram utilizados testes não-paramétricos de KruskalWallis, tomando-se, como tratamentos, as combinações entre os níveis de cada fator e os níveis de cada categoria, com o objetivo de detectar a existência de possíveis interações significativas. Os resultados obtidos, em termos dos p-valores, revelaram que não houve valor significativo $(\mathrm{p}<0,05)$.

Tais resultados indicam que os provadores portaram-se de modo homogêneo em cada um dos fatores considerados. Em outras palavras, não houve diferença significativa entre as preferências de homens e mulheres quanto às árvores, aos arbustos, aos bambus e assim por diante, de modo análogo para os demais fatores. Assim, houve evidências de que a deficiência, por si só, esteve acima desses fatores e que o escore atribuído deve-se, exclusivamente, ao caráter afetivo motivado pelas características das plantas avaliadas.

\subsection{Espécies arbóreas}

$\mathrm{Na}$ análise das preferências afetivas relacionadas à categoria arbórea, consideraram-se os fatores circunferência à altura do peito (CAP) e textura do caule.

Com base nos resultados obtidos, alguns fatos podem ser considerados na seleção de espécies arbóreas a fim de elaborar projetos de futuros jardins sensoriais, conforme segue:

- Embora a guaraiúva e o resedá tenham sido avaliados, com unanimidade, pelo escore máximo, não se pode perder de vista que, pelo teste de Dunn, os escores médios de tais plantas não apresentaram diferença significativa, em relação às médias dos escores atribuídos às seguintes plantas: melaleuca, pau-mulato, ipê-roxo e pau-ferro.

- Não houve unanimidade de escores máximos em nenhuma das palmeiras. Além disso, o desvio-padrão ${ }^{5}$, que considera todos os escores atribuídos a uma determinada planta, e, também, a amplitude total, que descreve a diferença entre o maior e o menor de tais escores, apresentam os dois dentre os três maiores valores: butiá $(2,8$ e 6,0) e livistona $(2,0$ e 6,0), evidenciando fortes discrepâncias entre as opiniões dos provadores. Na mesma direção, inclui-se, entre as árvores, a peroba $(2,4$ e 6,0$)$. - Os bambus, mesmo em pequeno número no experimento, revelaram muito bem aceitos pelos provadores.

- As palmeiras receberam o menor escore médio, dentre os três grupos arbóreos estudados, mesmo apresentando CAP médio $(0,90 \mathrm{~m})$ menor que o CAP médio das árvores $(1,90 \mathrm{~m})$. Tal fato parece sugerir, conforme verificado pelos componentes principais, que a característica textura da casca foi mais importante que o CAP, na escolha afetiva dos provadores.

- Por outro lado, houve evidências, nas condições do experimento realizado, que a palmeira butiá, por apresentar escore médio extremamente baixo, além de cajá-mirim e tipuana, não foram aprovadas pelos deficientes visuais. Por sua vez, em decorrência da grande variabilidade apresentada nos escores da peroba e da livistona, sugerese que outros experimentos sejam realizados com elas e, naturalmente, que sua inclusão em jardins sensoriais seja considerada com cautela.

\subsection{Espécies arbustivas}

$\mathrm{Na}$ análise das preferências afetivas relacionadas à categoria arbustiva, foram considerados os seguintes fatores: porte do arbusto e tipo, textura e tamanho das folhas. Observou-se que oito arbustos receberam o escore máximo de todos os provadores, sendo classificados unanimemente com a média 9,0: árvore-da-felicidade, buxinho, congea, cróton, dracena-rubra, goimbê, pata-deelefante e pleomele.

Um fato a ser considerado é que tais arbustos não apresentaram diferença significativa, pelo teste de Dunn, com a nolina, o manacá, a dracena young, a cheflera, a clúsia, a dracena-pau-d'água e a zâmia. Nesse contexto, esses 15 arbustos podem ser classificados como preferidos pelos provadores.

De modo análogo, observa-se, também pelo teste de Dunn, que dois arbustos mereceram escores médios significativamente menores que os demais: a palmeira ráfis $(5,3)$ e a acalifa $(7,4)$, refletindo pequeno índice de afetividade por parte dos provadores. Naturalmente, tal

$\left(^{3}\right)$ Desvio-padrão: medida estatística utilizada para quantificar a dispersão dos dados. Maiores valores de desvio-padrão indicam conjuntos de dados mais dispersos ou menos homogêneos. 
evidência deve ser considerada em futuras escolhas para a construção de jardins sensoriais.

\subsection{Espécies herbáceas}

$\mathrm{Na}$ análise das preferências afetivas, relacionadas à categoria herbácea, foram considerados os fatores porte da herbácea e tipo, textura e tamanho das folhas, além da ocorrência de flores e/ou inflorescência. Em relação às herbáceas, observou-se que nove espécies receberam o escore máximo de todos os provadores, sendo classificadas unanimemente com a média 9,0: agapanto, amarílis, calanchoe, "new" calanchoe, chifre-de-veado, cinerária, dinheiro-em-penca, gerânio e hemerocale.

De modo análogo aos parágrafos anteriores, tais herbáceas não apresentaram diferença significativa, pelo teste de Dunn, apresentando os seguintes resultados: agave $(8,5)$, begônia $(8,6)$, curculigo $(8,6)$, lírio-da-paz $(8,6)$ e nephrolepis $(8,6)$. Nesse contexto, essas 14 herbáceas podem ser classificadas como as preferidas pelos provadores.

De modo semelhante, observa-se, também pelo teste de Dunn, que a cavalinha recebeu escore médio 5,4, significativamente menor que os demais. Assim, a cavalinha motivou um pequeno índice de afetividade por parte dos provadores. Naturalmente, tal evidência deve ser considerada em futuras escolhas para a construção de jardins sensoriais.

O teste de medianas detectou diferença significativa ( $p<0,001)$ entre os escores atribuídos, em favor das plantas contempladas com flores ou inflorescências durante a avaliação. Observou-se a forte diferença entre os escores médios mínimos atribuídos às herbáceas com $(8,1)$ e sem $(5,4)$ flores ou inflorescências.

Há evidências, neste estudo, de que a presença do aspecto floral tenha influenciado positivamente os valores afetivos dos provadores, a bem da verdade, tão especiais. Exceções foram observadas no antúrio e na estrelícia, provavelmente por apresentarem folhas médias e grandes, respectivamente, ou características específicas em suas flores.

\subsection{Espécies gramíneas para relvados}

$\mathrm{Na}$ análise das preferências afetivas, relacionadas à categoria gramínea, foram considerados os fatores textura e tamanho das folhas. Observou-se que a grama coreana recebeu o escore máximo de todos os provadores, sendo classificada, unanimemente, com a média 9,0, e diferindo significativamente das demais gramíneas. O segundo grupo ficou constituído pelas gramas esmeralda $(7,9)$, santoagostinho $(7,2)$ e batatais $(6,3)$.

\subsection{Espécies vegetais que agregaram maiores valores afetivos aos provadores}

A tabela 3 fornece a relação das espécies destacadas, por agregar os maiores valores afetivos dentre as incluídas no experimento. Sua seleção foi realizada, tomando-se como critério escolher "as melhores", segundo os testes de Dunn, as que apresentaram amplitude total menor ou igual a 4,0 .

\section{CONCLUSÕES}

Não houve diferença significativa entre os níveis dos fatores sexo, idade, grau e tempo da deficiência, no que se refere às preferências sobre as espécies vegetais dentro de cada categoria.

Constatou-se que, aos menores valores de CAP e à textura mais fina, foram associados os maiores escores médios, refletindo a preferência dos provadores por indivíduos com menores diâmetros e por espécies com texturas macias, lisas e muito lisas. Além disso, os resultados sugeriram que a característica textura da casca foi mais importante, do ponto de vista afetivo, que a circunferência do caule à altura do peito.

No que se refere aos arbustos testados, verificouse que o porte foi pouco importante e que a textura e o tamanho das folhas foram fatores preponderantes na discriminação das espécies, com ampla vantagem para as texturas macias.

De modo análogo às espécies arbustivas, as características mais importantes das herbáceas, do ponto de vista afetivo, foram a textura e o tamanho das folhas. As herbáceas contempladas com flores ou inflorescências, no momento da avaliação, receberam escores médios classificados entre os maiores do experimento, exceção feita ao antúrio e à estrelícia, provavelmente por apresentarem folhas médias e grandes, respectivamente, ou características específicas em suas flores.

\section{CONSIDERAÇÕES FINAIS}

Os estudos realizados durante o presente trabalho possibilitaram a obtenção de informações consistentes sobre a percepção tátil de espécies vegetais, comumente utilizadas em projetos de paisagismo por parte de deficientes visuais, estabelecendo suas preferências afetivas para plantas de grupos distintos da vegetação, a saber: árvores, palmeiras, bambus, espécies arbustivas, herbáceas e gramas destinadas à formação de relvados.

De acordo com os experimentos realizados, ficou evidente a preferência dos provadores por plantas com folhagens consideradas macias, cascas dos caules (incluindo colmos e estipes) com textura lisa, bem como por espécies de folhas pequenas e lisas. As plantas que apresentavam flores ou inflorescências, por ocasião dos testes de afetividade, também foram mais apreciadas pelos provadores, provavelmente por seu aspecto delicado.

Além disso, no decorrer dos testes, pôde-se constatar, pelo convívio com os provadores, várias manifestações e posicionamentos relevantes para este estudo, tais como:

- o desfrute de uma planta e o eventual aproveitamento de um jardim, como local de recreação, de lazer, de entretenimento e de aprendizado, pelos deficientes visuais, é tanto mais positivo quanto mais as espécies vegetais permitam sua completa compreensão tátil, sem oferecer riscos à integridade de suas mãos; além disso, as plantas tornam-se mais interessantes quando são mais facilmente decifráveis pelas suas dimensões ou suas características específicas;

- A intenção de reexploração, especialmente nas plantas consideradas interessantes e agradáveis ao tato, observada, por exemplo, durante os testes com o gerânio, em que os provadores passaram diversas vezes os dedos pelas suas 
folhas aveludadas e macias;

- os comentários efetuados pelos provadores, durante os testes, abordaram detalhes específicos das plantas, principalmente com relação às suas dimensões exageradas (caule da peroba, folhas do goimbê etc.), à textura agradável (casca lisa da guaraiúva), em oposição ao caráter de sensações desagradáveis, percebidas pelo toque em superfícies ásperas e formas pontiagudas (palmeira butiá, espada-de-são-jorge);

Durante os testes, observou-se, também, que alguns provadores buscavam a idéia de um todo, abraçando a planta, enquanto outros iniciavam sua avaliação pelos detalhes, procurando conhecer as dimensões, as formas e a textura das folhas para, depois, tentarem compreender o todo.

Todas essas constatações podem servir para embasar um plano de instalação de um jardim sensorial especialmente projetado para atender às pessoas portadoras de necessidades especiais. Assim, eles poderão vivenciar uma experiência incomum que, com certeza, contribuirá para aumentar sua percepção sobre o ambiente natural que os envolvem, possibilitando gratificantes momentos de lazer.

\section{REFERÊNCIAS}

ASSOCIAÇÃO BRASILEIRA DE NORMAS TÉCNICAS Análise sensorial dos alimentos e bebidas. Terminologia. São Paulo: ABNT, 1993. 1v. (BBR 12806).

CAPPELARI JUNIOR, L. Sistemática de plantas medicinais e aromáticas. Piracicaba: Esalq-USP/ Departamento de Ciências Biológicas. 2006. 164p.

CLAUSTRIAUX, J.J.; IEMMA, A. F. A propos des qualificatifs complet, orthogonal et équilibré en analyse de la variance. Notes de Statistique et Informatique, Gembloux, n.2, p.1-14, 1999.

CONOVER, W.J. Practical nomparametric statistics. $2^{\text {nd }}$. ed. New York: Wiley, 1980. 494p.

DUNN, O.J. Multiple contrasts using rank sums. Technometrics, Alexandria, n.6, p.241-252,1964.

FERRARI, F.; IEMMA, A.F. Um critério para seleção de variáveis na seleção de componentes principais. Revista de Matemática e Estatística, São Paulo. v.8, n.1, p.101113,1991

FERREIRA, V.L.P. Análise sensorial: testes discriminativos e afetivos. Campinas: SBCTA, 2000. 127p. (Manual: Série qualidade)

IEMMA, A.F. Que hipóteses estatísticas estamos através do SAS em presença de caselas vazias? Scientia Agricola, Piracicaba. v.52, n.2, p.210-220, 1995.
IEMMA, A.F.; CLAUSTRIAUX, J.J. Étude des hypothèses de l'analyse de la variance à deux critères de classification : 1'approche par 1'exemple. Notes de Statistique et Informatique, Gembloux, n.3, p.1-36, 1999.

INSTITUTO BRASILEIRO DE GEOGRAFIA E ESTATÍSTICA (IBGE). Recenseamento de $\mathbf{2 0 0 0}$. Disponível em: www.ibge.gov.br. Acesso em: 22 jan. 2007

JONES, L.V.; PERYAM, D.R.; THRUSTONE, L.L. Development of a scale for measuring soldieres food preferences. Food Research, Champaign, v.20, n.5, p.512$520,1955$.

LORENZI, H. Árvores brasileiras: manual de identificação e cultivo de plantas arbóreas nativas do Brasil. Nova Odessa: Plantarum, 1992. 352p.

LORENZI, H. Árvores brasileiras: manual de identificação e cultivo de plantas arbóreas nativas do Brasil. 2.ed. Nova Odessa: Plantarum, 2002. v.2. 368p.

LORENZI, H.; SOUZA, M.S. Plantas ornamentais no Brasil: arbustivas, herbáceas e trepadeiras. 3. ed. Nova Odessa: Plantarum, 2001. 1088p.

LORENZI, H.; SOUZA, M.S.; TORRES, M.A.V. Árvores exóticas no Brasil: madeireiras, ornamentais e aromáticas. Nova Odessa: Instituto Plantarum, 2003. 368p.

MEILGAARD, M. ; CIVILle, G.V. ; CARR, B.T. Sensory evaluation techniques. $3^{\text {rd }}$. ed. London : CRC Press, 1999. 387p.

PALM, R. L'analise em composantes principales: principes et applications. Notes de Statistique et Informatique, Gembloux, n.2, p.1-29, 1998.

PERYAM, D.R.; PILGRIM, F.J. Hedonic scale method of measuring food preferences. Food Technology, Chicago, v.11, n.9, p.9-14, 1957. Supplement.

SANTANA, D.G.; IEMMA, A.F. Funções estimáveis e hipóteses testáveis nos delineamentos ortogonais e parcialmente ortogonais com três fatores. Scientia Agricola, Piracicaba, v.59, n.2, p.295-302, 2002.

SAS INTITUT user's guide. Cary: SAS Institute, Release 6.11 of de SAS system. 2000. 946p.

STONE, H.; SIDEL, J.L. Sensory evaluation practices. $3^{\text {rd }}$. ed. San Diego: Elsevier, 2004. 377p.

UNISTAT 5.0 Statistical package for windows. User's guide. London: UNISTAT House, 2001. 801p.

ZAR, J. H. Biostatistical analysis. $4^{\text {th }}$. ed. London: Prentice-Hall, 1999. 732p. 
Tabela 1. Espécies vegetais utilizadas para os testes sensoriais de afetividade, do ponto de vista tátil

Table 1. Plant species designed for tactile sensorial tests of affectivity

\begin{tabular}{|c|c|}
\hline \multicolumn{2}{|c|}{ PORTE ARBÓREO (ARBOREAL PLANTS) } \\
\hline Nome científico (Scientific name) & Nome popular (Common name) \\
\hline \multicolumn{2}{|l|}{ Categoria: Árvores (Category: Trees) } \\
\hline Agathis robusta (C. Moore) F.M.Bailey & Kauri \\
\hline Aspidosperma cylindrocarpon M. Arg. & Peroba (poca) \\
\hline Caesalpinia ferrea Mart. ex Tul. var. Leiostachya & Pau-ferro \\
\hline Calycophyllum spruceanum Benth. & Pau-mulato \\
\hline Lafoensia glyptocarpa Koehne & Mirindiba \\
\hline Lagerstroemia indica Lam & Resedá \\
\hline Melaleuca lineariifolia Sm. & Melaleuca \\
\hline Pinus elliottii Engel. & Pinheiro \\
\hline Spondias lutea L. & Cajá-mirim \\
\hline Securinega guaraiuva Kuhlm & Guaraiúva \\
\hline Tabebuia heptaphylla (Vell.) Tol. & Ipê-roxo \\
\hline Tabebuia chrysotricha (Mart. ex DC.) Standl. & Ipê-amarelo-cascudo \\
\hline Tipuana tipu (Benth.) Kuntze & Tipuana \\
\hline \multicolumn{2}{|l|}{ Categoria : Palmeiras (Category: Palm trees) } \\
\hline Archontophoenix alexandrae (F.Muell.) H.Wendl. \& Drude & Palmeira seafórcia \\
\hline Butia eriospatha (Mart.ex Drude) Becc. & Palmeira butiá \\
\hline Cocos nucifera $\mathrm{L}$. & Coco-da-bahia \\
\hline Livistona decipiens Becc. & Palmeira livistona \\
\hline Rhapis excelsa (Thunb.) A. Henry ex Rehder & Palmeira-rápis \\
\hline \multicolumn{2}{|l|}{ Categoria : Bambus (Category: Bamboo) } \\
\hline Bambusa vulgaris "vittata” Schrad & Bambu-amarelo \\
\hline \multicolumn{2}{|c|}{ PORTE ARBUSTIVO (SHRUBS) } \\
\hline Nome científico (Scientific name) & Nome popular (Common name) \\
\hline \multicolumn{2}{|l|}{ Categoria: Arbustos (Category: Shrubs) } \\
\hline Acalypha wilkesiana Müll. Arg. & Acalifa \\
\hline Beaucarnea recurvata Lem. & Nolina \\
\hline Buxus sempervirens L. & Buxinho \\
\hline Brunfelsia uniflora (Pohl) D. Don & Manacá-de-cheiro \\
\hline Codiaeum variegatum (L.) A. Juss. & Cróton \\
\hline Clusia fluminensis Planch. \& Triana & Clúsia \\
\hline
\end{tabular}


PORTE ARBUSTIVO (SHRUBS) (continuação)

\begin{tabular}{ll}
\hline Nome científico (Scientific name) & Nome popular(Common name) \\
\hline Categoria: Arbustos (Category: Shrubs) & \\
\hline Congea tomentosa Roxb. & Congea \\
Cordyline terminalis (L.) Kunth & Dracena-rubra \\
Dracaena deremensis Engl. & Dracena “young" \\
Dracaena fragans L. Ker Grawl. & Dracena-pau-d'água \\
Eugenia sprengelli DC. & Eugênia \\
Nandina domestica Thunb. & Nandina \\
Philodendron bipinnatifidum Schott & Goimbê \\
Pleomele reflexa N.E. Br. & Pleomele \\
Polyscias fruticosa (L.) Harms & Árvore-da-felicidade \\
Portulacaria afra Jacq. & Pata-de-elefante \\
Raphis excelsa (Thunb.) A. Henry ex Rehder & Palmeira-ráfis \\
Schefflera arboricola (Hayata) Merr. & Cheflera pequena \\
Zamia pumila L. & Zâmia \\
\hline
\end{tabular}

\section{PORTE HERBÁCEO (HERBACEOUS PLANTS)}

\begin{tabular}{|c|c|}
\hline Nome científico (Scientific name) & Nome popular (Common name) \\
\hline \multicolumn{2}{|l|}{ Categoria: Herbáceas (Category: Herbs) } \\
\hline Agapanthus africanus (L.) Hoffsgg & Agapanto \\
\hline Agave attenuata Salm-Dyck & Agave-dragão \\
\hline Anthurium andraeanum Linden & Antúrio-de-flor \\
\hline Anthurium spectabile Schott & Antúrio-taioba \\
\hline Begonia cucullata Willd. & Begônia-cerosa \\
\hline Calathea louisae Gagnep. & Calatéia \\
\hline Callisia repens (Jacq.) L. & Dinheiro-em-penca \\
\hline Chlorophytum comosum (Thunb.) Jacques & Clorófito, gravatinha \\
\hline Curculigo capitulata (Lour.) Kuntze & Curculigo \\
\hline Equisetum giganteum L. & Cavalinha \\
\hline Hemerocallis flava $\mathrm{L}$. & Hemerocalis \\
\hline Hippeastrum hybridum Hort. & Amarílis \\
\hline Iresine herbstii Hook. & Iresine \\
\hline Liriope muscari (Decne.) L.H. Bailey & Barba-de-serpente \\
\hline Kalanchöe blossfeldiana Poelln. & Calanchoe \\
\hline Kalanchöe manginii Raym.-Hamet \& H. Perrier & New calanchoe \\
\hline Maranta leuconeura E. Morren "Erythroneura" & Maranta \\
\hline Nephrolepis pectinata (Willd.) Schott & Samambaia-paulista \\
\hline
\end{tabular}




\begin{tabular}{|c|c|}
\hline \multicolumn{2}{|c|}{ PORTE HERBÁCEO (HERBACEOUS PLANTS) (continuação) } \\
\hline Nome científico (Scientific name) & Nome popular (Common name) \\
\hline \multicolumn{2}{|l|}{ Categoria: Herbáceas (Category: Herbs) } \\
\hline Ophiopogon japonicus (L.f.) Ker Gawl. & Grama-preta \\
\hline Pelargonium hortorum L.H. Bailey & Gerânio \\
\hline Pilea involucrata (Sims) Urb. & Pilea \\
\hline Platycerium bifurcatum C. Chr. & Chifre-de-veado \\
\hline Polypodium punctatum Thunb. & Ninho-de-passarinho \\
\hline Rhipsalis baccifera (J.S. Muell.) Stearn & Ripsális \\
\hline Russelia equisetiformis Schltdl. \& Cham. & Russélia \\
\hline Sansevieria cylindrica Bojer & Lança-são-jorge-cilíndrica \\
\hline Sansevieria trifasciata Pain "Hahnii" & Espada-são-jorge (anã) \\
\hline Sansevieria trifasciata var.laurentii (De Wild.)N.E. Br & Espada-são-jorge \\
\hline Schizocentron elegans Meissn. & Quaresmeira-rasteira \\
\hline Selaginella kraussiana (Kunze) A. Braun & Selaginela, musgo-tapete \\
\hline Senecio douglasii DC. & Cinerária \\
\hline Spathiphyllum cannifolium (Dryand.) Schott & Lírio-da-paz \\
\hline Strelitzia reginae Aiton & Estrelícia \\
\hline Vriesea hybrida Hort. & Bromélia \\
\hline \multicolumn{2}{|c|}{ PORTE RASTEIRO (GRASSES) } \\
\hline Nome científico (Scientific name) & Nome popular (Common name) \\
\hline \multicolumn{2}{|l|}{ Categoria: Gramas (Category: Grasses) } \\
\hline Paspalum notatum Flüggé & Batatais \\
\hline Stenotaphrum secundatum (Walter) Kuntze & Santo-agostinho \\
\hline Zoysia japonica Steud. & Esmeralda \\
\hline Zoysia tenuifolia Willd. \& Thiele & Coreana \\
\hline
\end{tabular}

Tabela 2. Conceitos afetivos e escores correspondentes

Table 2. Affective concepts and corresponding scores

\begin{tabular}{lccc}
\hline \multicolumn{1}{c}{\begin{tabular}{c}
\multicolumn{1}{c}{$\begin{array}{c}\text { Conceitos afetivos } \\
\text { (Affective Concepts) }\end{array}$} \\
Gostei muito (Really liked it)
\end{tabular}} & $\begin{array}{c}\text { Classes numéricas } \\
\text { (Grade categories) }\end{array}$ & $\begin{array}{c}\text { Pontos médios } \\
\text { (Average Grades) }\end{array}$ & $\begin{array}{c}\text { Escores } \\
\text { (Scores) }\end{array}$ \\
Gostei (Liked it) & $8,0-10,0$ & 9,0 & 9,0 \\
$\begin{array}{l}\text { Não gostei nem desgostei } \\
\text { Neither liked it or disliked it) }\end{array}$ & $6,0-8,0$ & 7,0 & 7,0 \\
Desgostei (Disliked it) & $4,0-6,0$ & 5,0 & 5,0 \\
Desgostei muito (Really disliked it) & $2,0-4,0$ & 3,0 & 3,0 \\
\hline
\end{tabular}


Tabela 3. Espécies vegetais que receberam os maiores escores médios dos provadores

Table 3. Plant species that received the highest average scores from the testers

\begin{tabular}{|c|c|c|c|}
\hline $\begin{array}{l}\text { Espécies } \\
\text { (Species) }\end{array}$ & & $\begin{array}{c}\text { Escore médio } \\
\text { (Average Score) }\end{array}$ & $\begin{array}{l}\text { Desvio-padrão } \\
\text { Standard Deviation }\end{array}$ \\
\hline \multicolumn{4}{|l|}{ Arbóreas - árvores (Arboreous trees) } \\
\hline \multicolumn{4}{|l|}{ Arbóreas (Arboreous) } \\
\hline Securinega guaraiuva Kuhlm & Guaraiúva & 9,0 & 0,0 \\
\hline Tabebuia heptaphylla (Vell.) Tol. & Ipê-roxo & 8,0 & 1,3 \\
\hline Melaleuca lineariifolia SM. & Melaleuca & 8,8 & 0,7 \\
\hline $\begin{array}{l}\text { Caesalpinia ferrea Mart. ex Tul. var. } \\
\text { Leiostachya }\end{array}$ & Pau-ferro & 7,7 & 1,6 \\
\hline Calycophyllum spruceanum Benth. & Pau-mulato & 8,5 & 0,9 \\
\hline Lagerstroemia indica Lam & Resedá & 9,0 & 0,0 \\
\hline \multicolumn{4}{|l|}{ Arbóreas - palmeiras (palm trees) } \\
\hline Cocos nucifera $\mathrm{L}$. & Coco-da-bahia & 8,4 & 1,3 \\
\hline Syagrus romanzoffiana Glas. & Jerivá & 8,3 & 1,0 \\
\hline $\begin{array}{l}\text { Archontophoenix alexandrae } \quad \text { (F.Muell.) } \\
\text { H.Wendl. \& Drude }\end{array}$ & Seafórcia & 8,3 & 1,0 \\
\hline \multicolumn{4}{|l|}{ Arbóreas - bambus (bamboos) } \\
\hline Bambusa vulgaris "vittata” Schrad & Bambu-amarelo & 9,0 & 0,0 \\
\hline Dendrocalamus giganteus (Wall) Munro. & Bambu-gigante & 8,6 & 1,0 \\
\hline \multicolumn{4}{|l|}{ Arbustivas (Shrubs) } \\
\hline Polyscias fruticosa (L.) Harms & $\begin{array}{l}\text { Árvore-da- } \\
\text { felicidade }\end{array}$ & 9,0 & 0,0 \\
\hline Beaucarnea recurvata Lem. & Nolina & 8,8 & 0.8 \\
\hline Buxus sempervirens L. & Buxinho & 9,0 & 0,0 \\
\hline Schefflera arboricola (Hayata) Merr. & Cheflera & 8,6 & 1,0 \\
\hline Clusia fluminensis Planch. \& Triana & Clúsia & 8,6 & 1,0 \\
\hline Congea tomentosa Roxb. & Congea & 9,0 & 0,0 \\
\hline Codiaeum variegatum (L.) A. Juss. & Cróton & 9,0 & 0,0 \\
\hline Dracaena fragans L. Ker Grawl. & $\begin{array}{l}\text { Dracena-pau- } \\
\text { d'água }\end{array}$ & 8,6 & 1,0 \\
\hline Cordyline terminalis (L.) Kunth & Dracena-rubra & 9,0 & 0,0 \\
\hline Dracaena deremensis Engl. & Dracena (young) & 8,8 & 0.8 \\
\hline Philodendron bipinnatifidum Schott & Goimbê & 9,0 & 0,0 \\
\hline Brunfelsia uniflora (Pohl) D. Don & $\begin{array}{l}\text { Manacá-de- } \\
\text { cheiro }\end{array}$ & 8,8 & 0.8 \\
\hline
\end{tabular}




\begin{tabular}{|c|c|c|c|}
\hline $\begin{array}{l}\text { Espécies } \\
\text { (Species) }\end{array}$ & & $\begin{array}{l}\text { Escore médio } \\
\text { (Average Score) }\end{array}$ & $\begin{array}{l}\text { Desvio-padrão } \\
\text { Standard Deviation }\end{array}$ \\
\hline Portulacaria afra Jacq. & Pata-de-elefante & 9,0 & 0,0 \\
\hline Pleomele reflexa N.E. Br. & Pleomele & 9,0 & 0,0 \\
\hline Zamia pumila $\mathrm{L}$. & Zâmia & 8,5 & 1,1 \\
\hline \multicolumn{4}{|l|}{ Herbáceas (Herbaceous) } \\
\hline Agapanthus africanus (L.) Hoffsgg & Agapanto (Flores) & 9,0 & 0,0 \\
\hline Hippeastrum hybridum Hort. & $\begin{array}{l}\text { Amarílis } \\
\text { (Flores) }\end{array}$ & 9,0 & 0,0 \\
\hline Begonia cucullata Willd. & $\begin{array}{l}\text { Begônia } \\
\text { (Flores) }\end{array}$ & 8,6 & 0,8 \\
\hline Kalanchöe blossfeldiana Poelln. & $\begin{array}{l}\text { Calanchoe } \\
\text { (Flores) }\end{array}$ & 9,0 & 0,0 \\
\hline $\begin{array}{l}\text { Kalanchöe manginii Raym.-Hamet \& } \mathrm{H} \text {. } \\
\text { Perrier }\end{array}$ & Calanchoe (new) & 9,0 & 0,0 \\
\hline Platycerium bifurcatum C. Chr. & Chifre-de-veado & 9,0 & 0,0 \\
\hline Senecio douglasii $D C$. & Cinerária & 9,0 & 0,0 \\
\hline Curculigo capitulata (Lour.) Kuntze & Curculigo & 8,6 & 0,8 \\
\hline Callisia repens (Jacq.) L. & $\begin{array}{l}\text { Dinheiro-em- } \\
\text { penca }\end{array}$ & 9,0 & 0,0 \\
\hline Pelargonium hortorum L.H. Bailey & Gerânio (Flores) & 9,0 & 0,0 \\
\hline Hemerocallis flava $\mathrm{L}$. & $\begin{array}{l}\text { Hemerocalis } \\
\text { (Flores) }\end{array}$ & 9,0 & 0,0 \\
\hline Spathiphyllum cannifolium (Dryand.) Schott & $\begin{array}{l}\text { Lírio-da-paz } \\
\text { (Inflorescência) }\end{array}$ & 8,6 & 0,8 \\
\hline Nephrolepis pectinata (Willd.) Schott & $\begin{array}{l}\text { Nephrolepis } \\
\text { (samambaia- } \\
\text { paulista) }\end{array}$ & 8,6 & 0,8 \\
\hline \multicolumn{4}{|l|}{ Gramíneas (Grasses) } \\
\hline Zoysia tenuifolia Willd. \& Thiele & Grama coreana & 9,0 & 0,0 \\
\hline
\end{tabular}

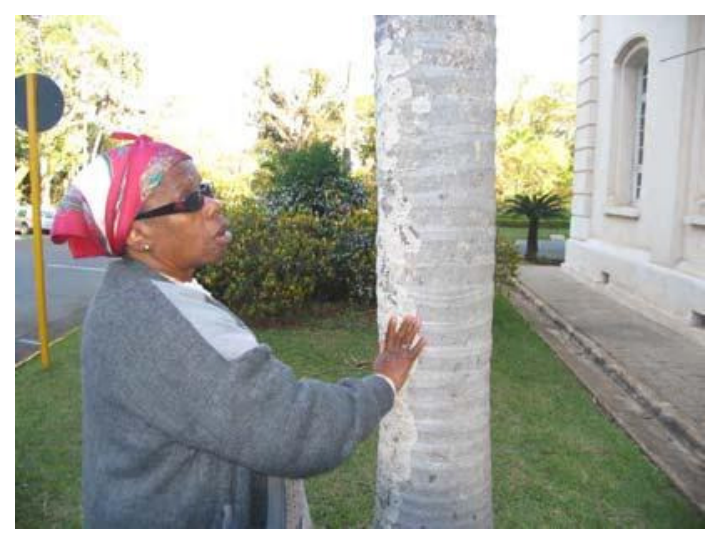

Figura 1. Teste sensorial realizado no Parque do campus Luiz de Queiroz (Piracicaba, SP) por um dos membros do grupo de provadores (Foto: José Flávio Machado Leão).

Figura 1. Sensorial test carried out at the Park on the Luiz de Queiroz campus (Piracicaba, SP) by one of the members of the tester group (Picture by: José Flávio Machado Leão) 